\title{
Phototriggers for nucleobases with improved photochemical properties
}

\author{
Toshiaki Furuta*, Takayoshi Watanabe, Satoshi Tanabe, Jun Sakyo, Chie Matsuba \\ Department of Biomolecular Science and Research Center for Materials with Iintegrated Properties, Toho \\ University, 2-2-1 Miyama, Funabashi, Chiba 274-8510, Japan \\ furuta@biomol.sci.toho-u.ac.jp
}

\section{Synthesis}

All reagents and solvents were purchased from commercial sources and used without further purification. Flash column chromatography was carried out using 43-60 mesh silica gel. NMR spectra were recorded on a JEOL GSX270 at $270 \mathrm{MHz}$ for ${ }^{1} \mathrm{H}$ and at $67 \mathrm{MHz}$ for ${ }^{13} \mathrm{C}$ and a Brucker Biospin Avance $300 \mathrm{M}$ at 300 $\mathrm{MHz}$ for ${ }^{1} \mathrm{H}$ and $75 \mathrm{MHz}$ for ${ }^{13} \mathrm{C}$ with a deuterated solvent as and TMS as an internal standard. IR spectra were recorded on a Thermo Nicolet Avatar 320 in ATR mode. Analytical HPLC was run on an Agilent HP 1100 system with DAD detection and preparative HPLC on a JASCO PU 9800 system with UV detection.

Synthesis of 4-N-(6-Bromo-7-hydroxycoumarin-4-ylmethoxycarbonyl)-2'-deoxycytidine (Bhcmoc-dC, 1a)
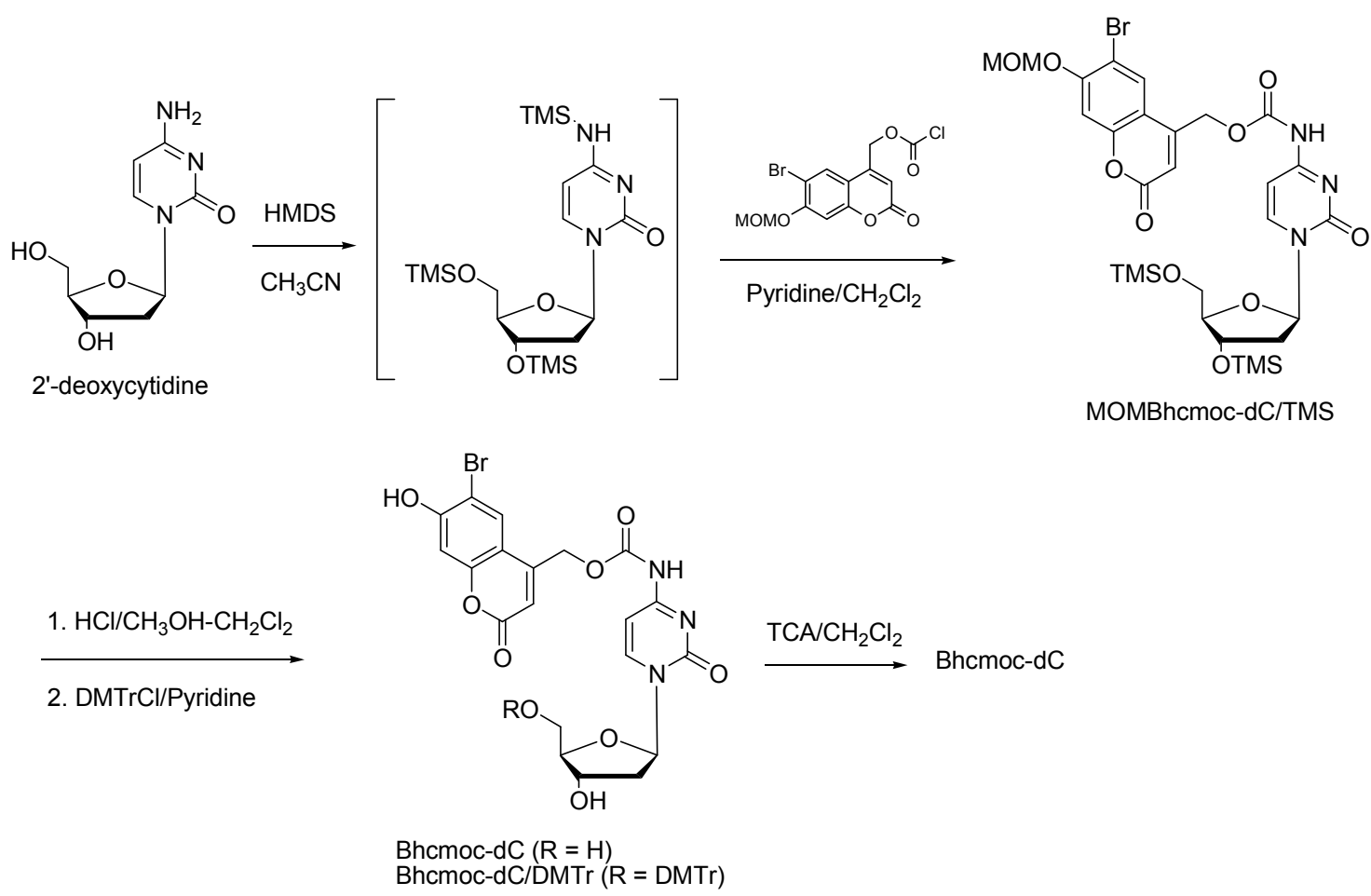

5'-O-(4,4'-dimethoxytrityl)-4- $N$-[(6-bromo-7-hydroxycoumarin-4-yl)methoxycarbonyl]-2'deoxycytidine (Bhemoc-dC/DMTr)

To a stirred suspension of the 2'-deoxycytidine hydrochloride (131.3 mg, $0.498 \mathrm{mmol})$ in acetonitrile (9 
$\mathrm{mL})$ was added $\operatorname{HMDS}(0.53 \mathrm{~mL}, 2.5 \mathrm{mmol})$ and the mixture was stirred at $60^{\circ} \mathrm{C}$ for $30 \mathrm{~min}$. The solvent was removed under vacuum. The residue was resuspended in dichloromethane $(9 \mathrm{~mL})$ and pyridine $(61 \mu \mathrm{L}$, $0.75 \mathrm{mmol})$ and stirred at $0^{\circ} \mathrm{C}$ for $5 \mathrm{~min}$. To this was added MOMBhcmoc- $\mathrm{Cl}(284.0 \mathrm{mg}, 0.752 \mathrm{mmol})$ and the stirring was continued at $0^{\circ} \mathrm{C}$ for $1 \mathrm{hr}$. The reaction mixture was diluted with dichloromethane and washed with $5 \% \mathrm{NaHCO}_{3}$. The organic layer was dried over $\mathrm{MgSO}_{4}$ and concentrated to give MOMBhcmoc-dC/TMS.

The crude product was dissolved in methanol $(10 \mathrm{~mL})$ and dichloromethane $(2 \mathrm{~mL})$. To the stirred solution were added three portions of conc. $\mathrm{HCl}(2 \mathrm{~mL}$ each) and the stirring was continued for $1 \mathrm{hr}$. The desired product 1a was precipitated with other unidentified materials and collected by filtration. The chromatographic purification of the product was difficult because of its low solubility in commonly used organic solvents, such as chloroform and ethyl acetate. Therefore, 1a was transformed into its 5'-protected derivative, Bhcmoc-dC/DMTr, which would be soluble in common organic solvents. After the chromatographic purification, the 5' protecting group was deprotected to give purified 1a. The followings are experimental details.

The collected precipitates were azeotropically dried with pyridine and re-dissolved in pyridine $(4 \mathrm{~mL})$. To the solution was added DMTr-Cl $(172.9 \mathrm{mg}, 0.510 \mathrm{mmol})$. The reaction mixture was stirred at $40^{\circ} \mathrm{C}$ for 90 min and quenched by adding $5 \% \mathrm{NaHCO}_{3}$. The mixture was diluted by chloroform. The organic layer was separated, dried over $\mathrm{MgSO}_{4}$ and concentrated under vacuum. Purification by column chromatography (19 $\mathrm{g}$ of silica gel 60, 4.3\% methanol in dichloromethane as an eluent) gave Bhcmoc-dC/DMTr (31.4 mg, 7.6\% yield from $\mathrm{dC}$ ).

${ }^{1} \mathrm{H}$ NMR $\left(\mathrm{CD}_{3} \mathrm{OD}\right) \delta 8.35(1 \mathrm{H}, \mathrm{d}, \mathrm{J}=7.5 \mathrm{~Hz}), 7.88(1 \mathrm{H}, \mathrm{s}), 7.20-7.40(9 \mathrm{H}, \mathrm{m}), 6.97(1 \mathrm{H}, \mathrm{d}, \mathrm{J}=7.5 \mathrm{~Hz})$, 6.83-6.88 $(5 \mathrm{H}, \mathrm{m}), 6.36(1 \mathrm{H}, \mathrm{s}), 6.15(1 \mathrm{H}, \mathrm{dd}, \mathrm{J}=5$ and $5 \mathrm{~Hz}), 5.41(2 \mathrm{H}, \mathrm{s}), 4.51(1 \mathrm{H}, \mathrm{m}), 4.08(1 \mathrm{H}, \mathrm{m})$, $3.77(3 \mathrm{H}, \mathrm{s}), 3.76(3 \mathrm{H}, \mathrm{s}), 3.45(1 \mathrm{H}, \mathrm{d}, \mathrm{J}=17 \mathrm{~Hz}), 3.42(1 \mathrm{H}, \mathrm{d}, \mathrm{J}=17 \mathrm{~Hz}), 2.56(1 \mathrm{H}$, ddd, $\mathrm{J}=13,6$ and 6 $\mathrm{Hz}), 2.30(1 \mathrm{H}, \mathrm{ddd}, \mathrm{J}=13,6$ and $5 \mathrm{~Hz})$

${ }^{13} \mathrm{C}$ NMR (DMSO-d 6 ) $\delta 162.57,159.70,158.11,157.49,154.04,153.76,149.77,144.57,135.36,135.21$, $129.73,128.49,127.88,127.71,126.78,113.22,110.26,108.67,106.24,103.14,94.03,85.96,85.87,85.70$, $69.29,62.87,62.05,54.99,40.75$. 


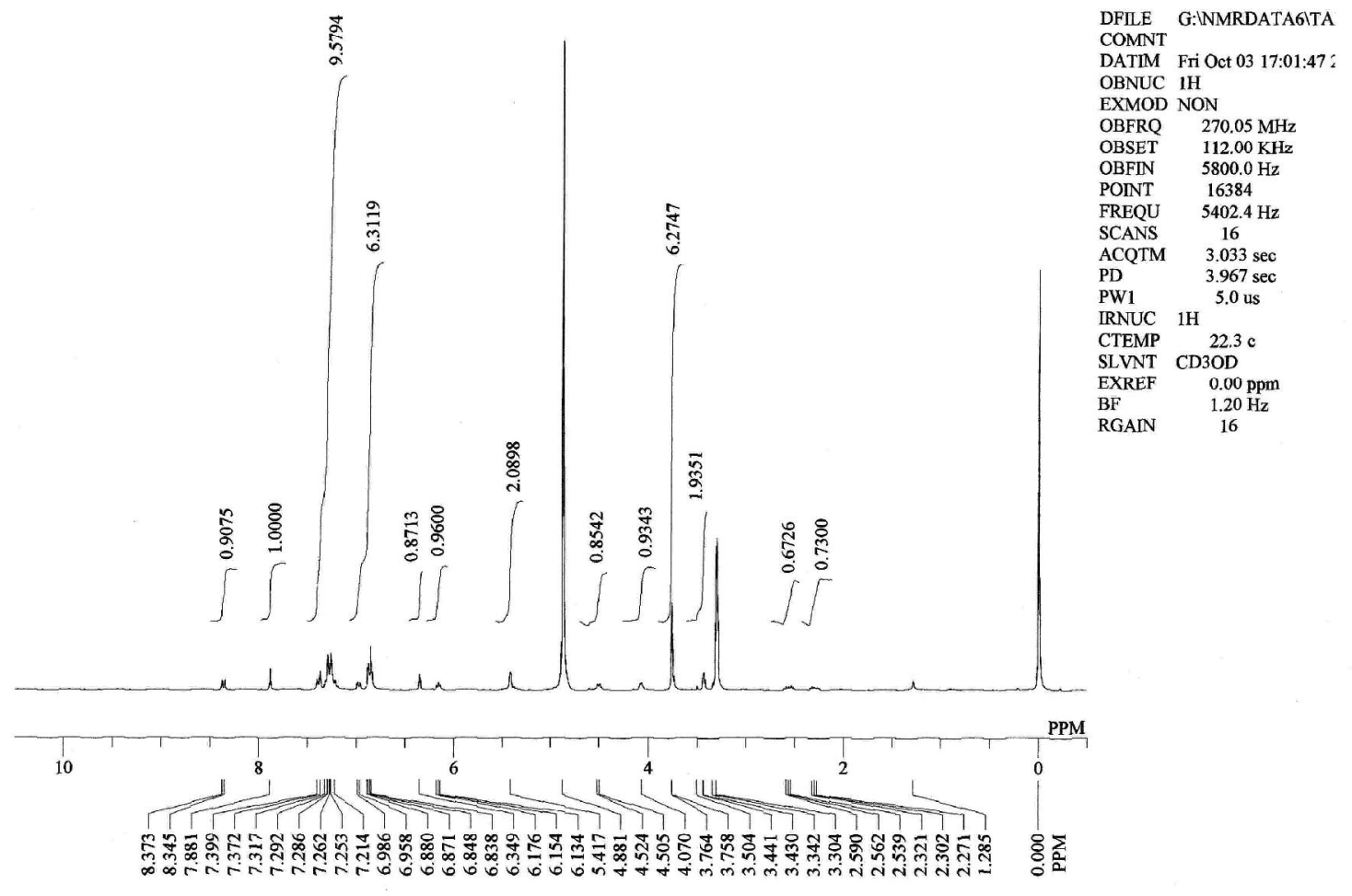

${ }^{1} \mathrm{H}$ NMR spectrum of Bhcmoc-dC/DMTr

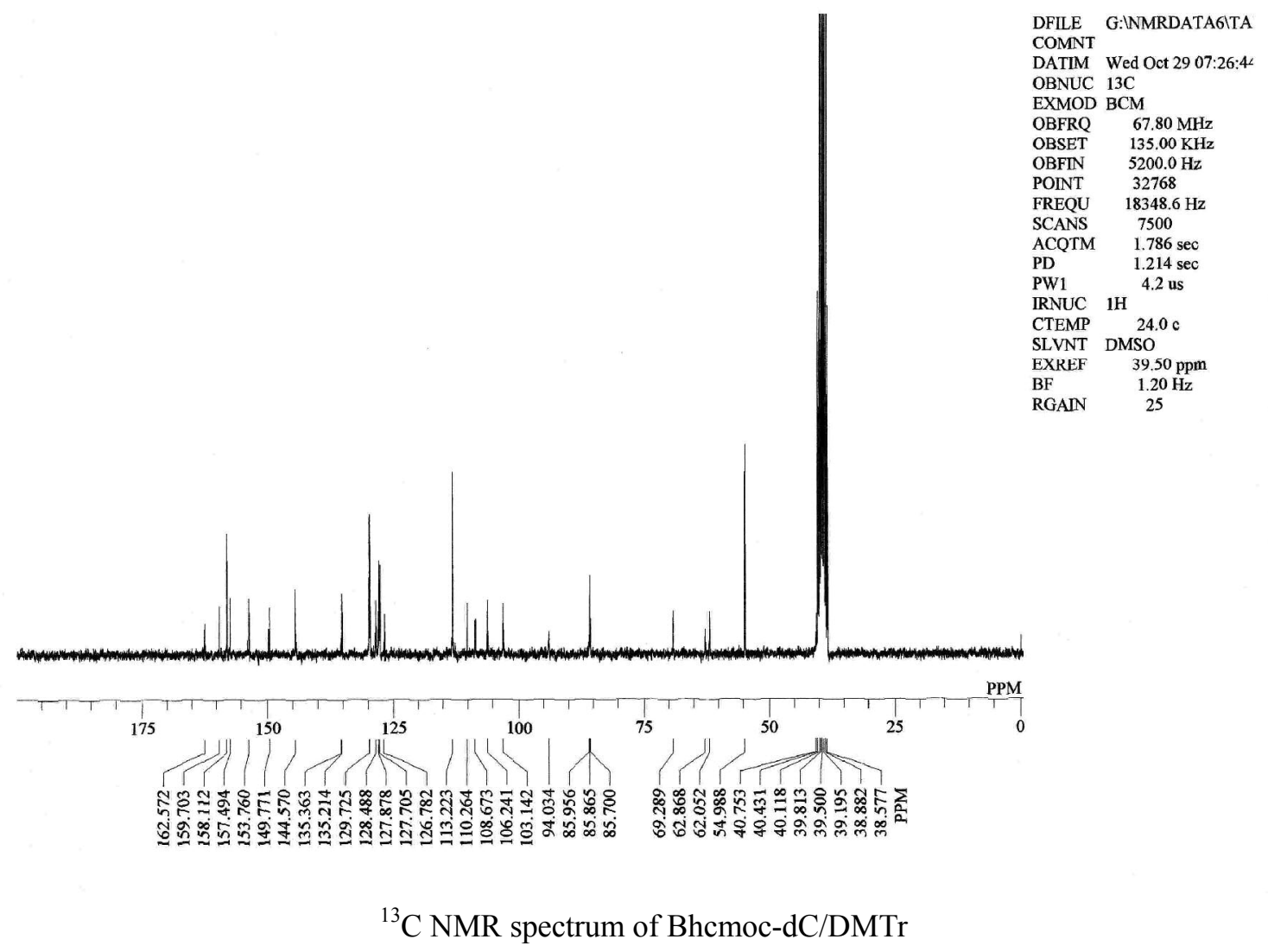


Bhcmoc-dC/DMTr $(17.1 \mathrm{mg}, 0.020 \mathrm{mmol})$ was dissolved in $0.05 \mathrm{M}$ TCA in dichloromethane $(0.05 \mathrm{~mL})$. The reaction mixture was stirred at r.t. for $30 \mathrm{~min}$. The solvents were removed under vacuum to yield 1a (7.5 mg, $0.014 \mathrm{mmol}, 67 \%$ yield).

${ }^{1} \mathrm{H}$ NMR (pyridine- $\left.\mathrm{d}_{5}\right) \delta 8.89(1 \mathrm{H}, \mathrm{d}, \mathrm{J}=7 \mathrm{~Hz}), 8.10(1 \mathrm{H}, \mathrm{s}), 7.44(1 \mathrm{H}, \mathrm{d}, \mathrm{J}=7 \mathrm{~Hz}), 6.91(1 \mathrm{H}, \mathrm{dd}, \mathrm{J}=5.5$ and $5.5 \mathrm{~Hz}), 7.03(1 \mathrm{H}, \mathrm{s}), 6.93(1 \mathrm{H}, \mathrm{dd}, \mathrm{J}=6.53(1 \mathrm{H}, \mathrm{s}), 5.68(2 \mathrm{H}, \mathrm{s}), 5.01(1 \mathrm{H}, \mathrm{ddd}, \mathrm{J}=5,5$ and $5 \mathrm{~Hz})$, $4.51(1 \mathrm{H}, \mathrm{ddd}, \mathrm{J}=3,3$ and $3 \mathrm{~Hz}), 4.26(1 \mathrm{H}, \mathrm{dd}, \mathrm{J}=12$ and $3 \mathrm{~Hz}), 4.18(1 \mathrm{H}, \mathrm{dd}, \mathrm{J}=12$ and $3 \mathrm{~Hz}), 2.96(1 \mathrm{H}$, ddd, $\mathrm{J}=13,6$ and $6 \mathrm{~Hz})), 2.65(1 \mathrm{H}$, ddd, $\mathrm{J}=13,6.5$ and $6.5 \mathrm{~Hz})$.

${ }^{13} \mathrm{C}$ NMR $\delta 164.4$ (s), 160.8 (s), 159.8 (s), 156.1 (s), 155.6 (s), 154.3 (s), 149.6 (s), 145.5 (d), 129.3 (d), $111.5(\mathrm{~s}), 110.7$ (d), $108.4(\mathrm{~s}), 104.6(\mathrm{~d}), 95.6(\mathrm{~d}), 89.8(\mathrm{~d}), 88.1$ (d), $71.1(\mathrm{~d}), 63.1(\mathrm{t}), 62.2(\mathrm{t}), 43.1(\mathrm{t})$ FT-IR (neat) 3000-3500 (brd), 1725, 1711, 1605, 1502, 1403, 1205, 1159, $1092 \mathrm{~cm}^{-1}$. 


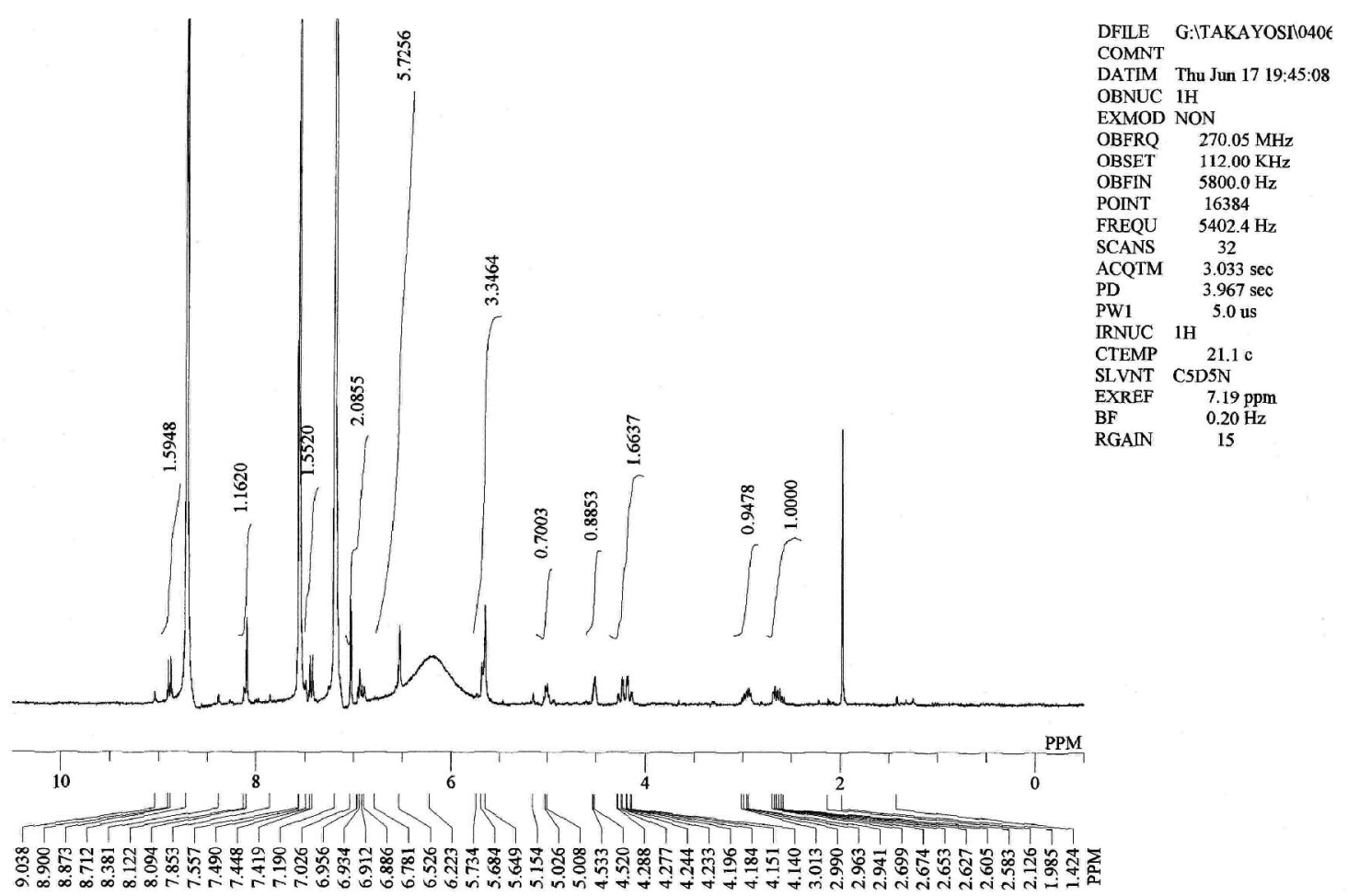

Bherase dC

${ }^{1} \mathrm{H}$ NMR spectrum of $\mathbf{1 a}$

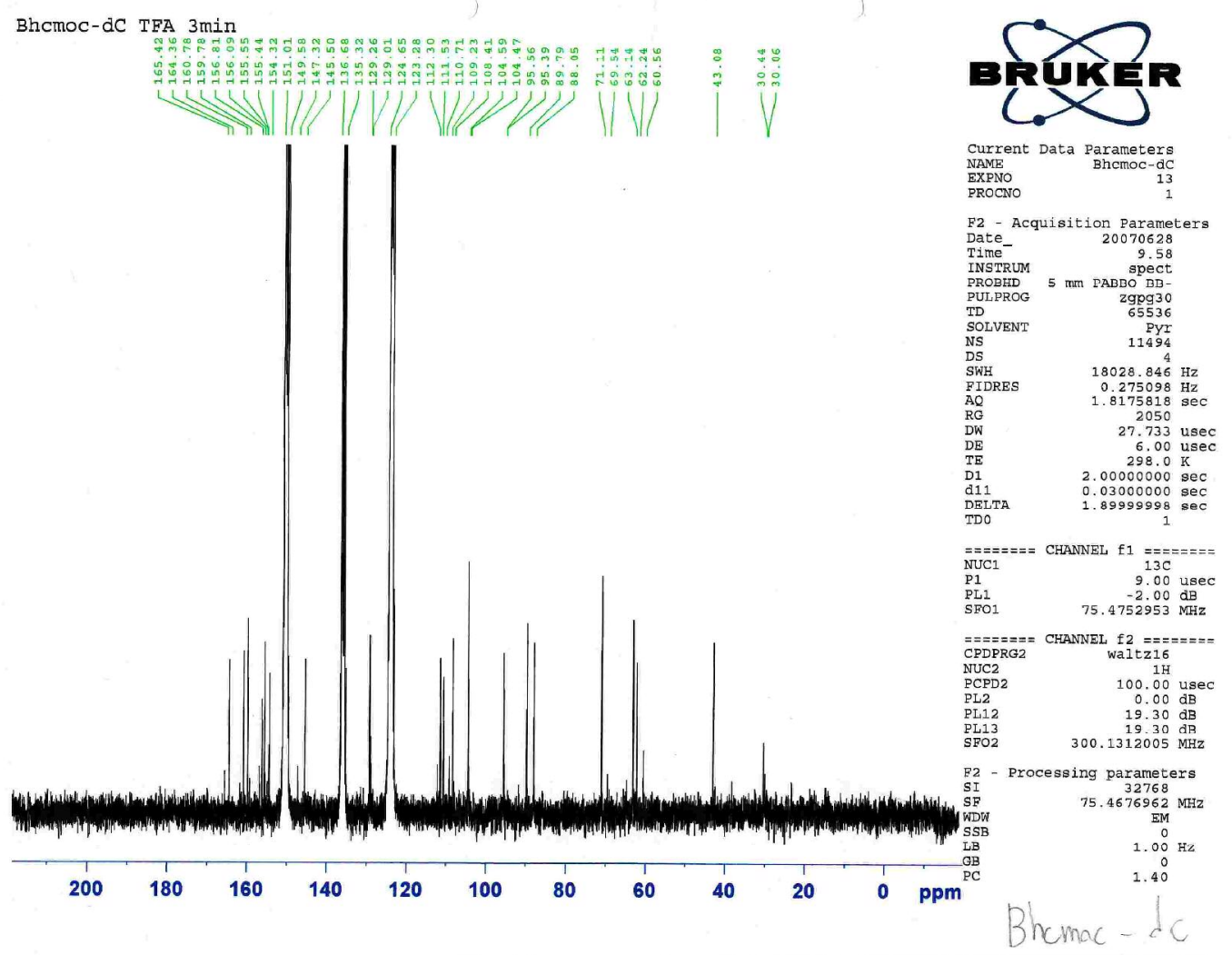

${ }^{13} \mathrm{C}$ NMR spectrum of $\mathbf{1 a}$ 
Synthesis of 4- $N$-[(6-Bromo-7-methoxycoumarin-4-yl)methoxycarbonyl]-2'-deoxycytidine

(Bmemoc-dC, 1b)<smiles>Cc1cc(=O)oc2cc(O)c(Br)cc12</smiles>

3

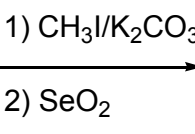

3) $\mathrm{NaBH}_{4}$<smiles>[R]c1cc(=O)oc2cc(OC)c(Br)cc12</smiles>

$4: \mathrm{R}=\mathrm{CH}_{3}$

$5: \mathrm{R}=\mathrm{CHO}$

$6: \mathrm{R}=\mathrm{CH}_{2} \mathrm{OH}$
$\mathrm{COCl}_{2} /$ hexane

THF-toluene<smiles>COc1ccc2c(C)cc(=O)oc2c1</smiles>

7: Bmcmoc-Cl<smiles>Nc1ccn(C2CC(O)C(CO)O2)c(=O)n1</smiles>

2'-deoxycytidine<smiles></smiles>

1b: Bmcmoc-dC

\section{6-Bromo-7-methoxy-4-methylcoumarin (4)}

To a stirred suspension of $530.8 \mathrm{mg}(2.08 \mathrm{mmol})$ of $\mathbf{3}$ and $301.5 \mathrm{mg}(2.18 \mathrm{mmol})$ of $\mathrm{K}_{2} \mathrm{CO}_{3}$ in acetone (10 $\mathrm{mL})$ was added $0.65 \mathrm{~mL}(10 \mathrm{mmol})$ of iodemethane. The reaction mixture was stirred at refluxed temperature for $7 \mathrm{hr}$ under Ar atmosphere. The reaction mixture was cooled to room temperature and the solvents were removed with rotovap. The residue was diluted with $\mathrm{CHCl}_{3}$, washed with $1 \mathrm{M} \mathrm{HCl}$ and dried over $\mathrm{MgSO}_{4}$. The solvents were removed with rotovap and high vacuum rotovap to give $518.4 \mathrm{mg}$ (1.93 mmol, $93 \%$ yield) of 4 .

${ }^{1} \mathrm{H}$ NMR $\delta 7.76(1 \mathrm{H}, \mathrm{s}), 6.84(1 \mathrm{H}, \mathrm{s}), 6.18(1 \mathrm{H}, \mathrm{s}), 3.97(3 \mathrm{H}, \mathrm{s}), 2.40(3 \mathrm{H}, \mathrm{s})$

${ }^{13} \mathrm{C}$ NMR $\delta 160.6,158.4,154.3,151.5,128.6,114.5,112.8,107.4,100.3,56.7,18.6$

\section{6-Bromo-4-formyl-7-methoxycoumarin (5)}

A mixture of 4 (390.8 mg, $1.45 \mathrm{mmol})$ and selenium dioxide $(177.8 \mathrm{mg}, 1.60 \mathrm{mmol})$ in chlorobenzene (15 $\mathrm{mL}$ ) was stirred at reflux temperature for $4 \mathrm{~d}$. The reaction mixture was cooled, filtered and evaporated. Purification by recrystallization from hexane and chloroform gave $361.3 \mathrm{mg}$ (1.28 mmol, 88\% yield) of compound 5 .

${ }^{1} \mathrm{H}$ NMR $\delta 10.05(1 \mathrm{H}, \mathrm{s}), 8.82(1 \mathrm{H}, \mathrm{s}), 6.89(1 \mathrm{H}, \mathrm{s}), 6.76(1 \mathrm{H}, \mathrm{s}), 3.99(3 \mathrm{H}, \mathrm{s})$.

${ }^{13} \mathrm{C}$ NMR $\delta 193.5,160.0,158.3,155.2,142.5,130.1,129.1,122.4,109.2,107.1,101.2,57.2$

\section{6-Bromo-4-hydroxymethyl-7-methoxycoumarin (6)}

To a stirred solution of $347.5 \mathrm{mg}(1.23 \mathrm{mmol})$ of 5 in ethanol $(4 \mathrm{~mL})$ and THF $(4 \mathrm{~mL})$ was added $47.3 \mathrm{mg}$ $(1.25 \mathrm{mmol})$ of sodium borohydride. The reaction mixture was stirred at room temperature for $4.5 \mathrm{hr}$. The reaction was quenched with methanol and the solvents were removed with rotovap. The residue was diluted 
with $\mathrm{CHCl}_{3}$, washed with $1 \mathrm{M} \mathrm{HCl}$ and dried over $\mathrm{Na}_{2} \mathrm{SO}_{4}$. The solvents were removed with rotovap and high vacuum rotovap to give $322.3 \mathrm{mg}$ ( $1.13 \mathrm{mmol}, 92 \%$ yield $)$ of $\mathbf{6}$.

${ }^{1} \mathrm{H}$ NMR $\delta 7.70(1 \mathrm{H}, \mathrm{s}), 6.86(1 \mathrm{H}, \mathrm{s}), 6.51(1 \mathrm{H}, \mathrm{t}, \mathrm{J}=1.6), 4.86(2 \mathrm{H}, \mathrm{s}), 3.97(3 \mathrm{H}, \mathrm{s})$.

${ }^{13} \mathrm{C}$ NMR $\delta 160.1,157.9,156.0,154.1,128.0,111.8,108.4,106.6,100.9,59.1,57.1$

\section{(6-Bromo-7-methoxycoumarin-4-yl)methoxycarbonyl chloride (Bmomoc-Cl, 7)}

In a glass pressure bottle (Hyperglasstor, THG-A2, TAIATSU Techno) $1.506 \mathrm{~g}$ (5.08 mmol) of triphosgene was placed. To this $198.4 \mathrm{mg}(0.49 \mathrm{mmol})$ of aliquat ${ }^{\mathrm{R}} 336 \mathrm{in} \mathrm{n}$-hexane $(4 \mathrm{~mL})$ was added. The mixture was stirrer at room temperature for $1 \mathrm{~d}$ to generate phosgene. To a stirred solution of $143.1 \mathrm{mg}(0.51 \mathrm{mmol})$ of 6 in toluene $(2 \mathrm{~mL})$ and THF $(2 \mathrm{~mL})$ was added the phosgene in n-hexane $(4 \mathrm{~mL})$. The reaction mixture was stirred at $0^{\circ} \mathrm{C}$ for $2.5 \mathrm{hr}$. The solvents were removed under reduced pressure. The residual solid was washed with $\mathrm{CHCl}_{3}$ and $n$-hexane $(1 / 1 \mathrm{v} / \mathrm{v})$ twice to give $124.9 \mathrm{mg}(0.36 \mathrm{mmol}, 71 \%$ yield $)$ of 7.

${ }^{1} \mathrm{H}$ NMR $\delta 7.63(1 \mathrm{H}, \mathrm{s}), 6.89(1 \mathrm{H}, \mathrm{s}), 6.42(1 \mathrm{H}, \mathrm{s}), 5.41(2 \mathrm{H}, \mathrm{s}), 3.99(3 \mathrm{H}, \mathrm{s})$.

${ }^{13} \mathrm{C}$ NMR $\delta 159.6,159.1,154.7,153.6,145.5,127.3,112.0,110.8,108.2,100.7,67.1,56.9$

\section{4- $N$-[(6-Bromo-7-methoxycoumarin-4-yl)methoxycarbonyl]-2'-deoxycytidine (Bmomoc-dC, 1b)}

The reported procedure for the preparation of NVOC- $\mathrm{dC}^{1}$ was slightly modified and applied to this compound. The 2'-deoxycytidine hydrochloride was dried by azeotropic removal of water with anhydrous pyridine. To a stirred suspension of the 2'-deoxycytidine hydrochloride (299.3 $\mathrm{mg}, 1.14 \mathrm{mmol})$ in anhydrous pyridine $(10 \mathrm{~mL})$ was added $\mathrm{TMSCl}(0.73 \mathrm{~mL}, 5.71 \mathrm{mmol})$ and the mixture was stirred at room temperature for $1 \mathrm{hr}$. To this was added Bmcmoc-Cl (465.6 mg, $1.34 \mathrm{mmol})$ and the stirring was continued for $14 \mathrm{hr}$. The reaction was quenched by adding water/methanol $(1 / 1,10 \mathrm{~mL})$ and the stirring was continued for $10 \mathrm{~min}$. The solvents were removed under reduced pressure. The residue was resuspended in dichloromethane and vigorously stirred with sat. $\mathrm{NaHCO}_{3}$ solution. The aqueous layer was separated and extracted with pyridine/dichloromethane (10/1) for three times. The combined organic layer was dried over $\mathrm{Na}_{2} \mathrm{SO}_{4}$, filtered and concentrated under reduced pressure to give $\mathbf{1 b}$ (276.3 mg, $0.510 \mathrm{mmol}, 45.1 \%$ yield). ${ }^{1} \mathrm{H}$ NMR (pyridine- $\left.\mathrm{d}_{5}\right) \delta 8.89(1 \mathrm{H}, \mathrm{d}, \mathrm{J}=7.5 \mathrm{~Hz}), 8.06(1 \mathrm{H}, \mathrm{s}), 7.42(1 \mathrm{H}, \mathrm{d}, \mathrm{J}=7.5 \mathrm{~Hz}), 7.00(1 \mathrm{H}, \mathrm{s}), 6.93$ $(1 \mathrm{H}, \mathrm{dd}, \mathrm{J}=6.5$ and $6.5 \mathrm{~Hz}), 6.59(1 \mathrm{H}, \mathrm{s}), 5.65(2 \mathrm{H}, \mathrm{s}), 5.01(1 \mathrm{H}, \mathrm{m}, 4.52(1 \mathrm{H}, \mathrm{m}), 4.24(1 \mathrm{H}, \mathrm{dd}, \mathrm{J}=12$ and $2 \mathrm{~Hz}), 4.18(1 \mathrm{H}, \mathrm{dd}, \mathrm{J}=12$ and $2 \mathrm{~Hz}), 3.80(3 \mathrm{H}, \mathrm{s}), 2.94(1 \mathrm{H}, \mathrm{ddd}, \mathrm{J}=10,5$ and $5 \mathrm{~Hz}), 2.60(1 \mathrm{H}$, ddd, $\mathrm{J}=10,5$ and $5 \mathrm{~Hz})$

${ }^{13} \mathrm{C}$ NMR (pyridine-d $\mathrm{d}_{5}$ ) $\delta 163.9$ (s), 160.1 (s), 159.0 (s), 155.5 (s), 155.2 (s), 153.9 (s), 149.1 (s), 140.0 (d), 128.5 (d), 112.0 (s), 111.3 (d), 107.8 (s), 101.2 (d), 95.0 (d), 89.3 (d), 87.6 (d), 70.7 (d), $62.6(\mathrm{t}), 61.8$ (t), $56.9(\mathrm{q}), 42.7(\mathrm{t})$

FT-IR (neat) 3300-3500 (brd), 3040, 1734, 1702, 1692, 1649, 1643, 1601, 1556, 1501, 1276, 1209, 1160, $1098 \mathrm{~cm}^{-1}$.

ESI-MS (positive) $\mathrm{m} / \mathrm{z} 538\left(\mathrm{C}_{21} \mathrm{H}_{20}{ }^{79} \mathrm{BrN}_{3} \mathrm{O}_{9}+\mathrm{H}^{+}\right), 540\left(\mathrm{C}_{21} \mathrm{H}_{20}{ }^{81} \mathrm{BrN}_{3} \mathrm{O}_{9}+\mathrm{H}^{+}\right)$ 


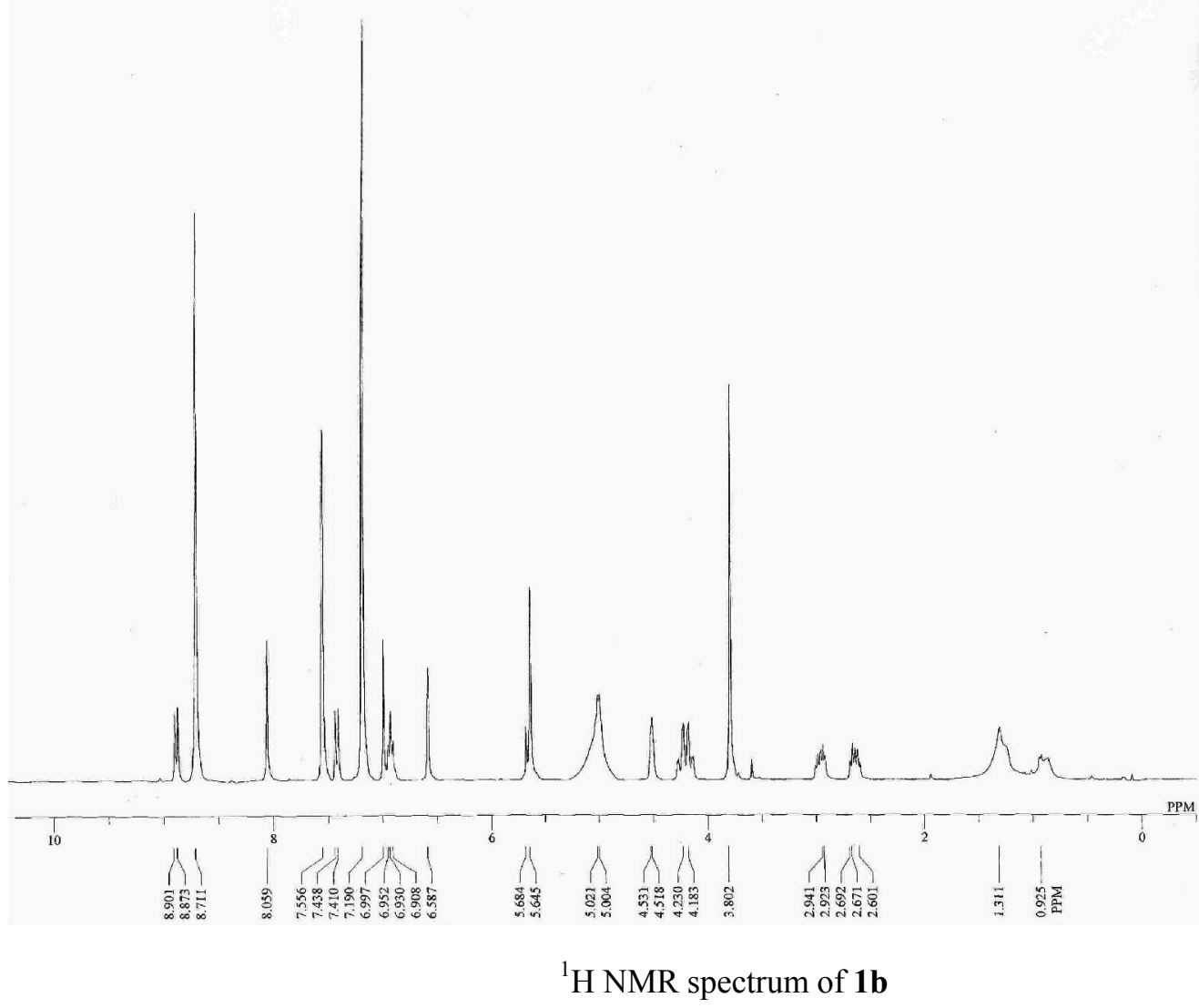

DFILE CIWINNMRICOMMON D

COMNT Wed Oct 22 23:16:04 2003

DATIM Wed

OBFRQ $270.05 \mathrm{MHz}$

$\begin{array}{ll}\text { OBSET } & 112.00 \mathrm{KHz} \\ \text { OBFIN } & 58000.0 \mathrm{~Hz}\end{array}$

\begin{tabular}{ll} 
POINT & 16384 \\
FREOU & $\$ 402.4$ \\
\hline
\end{tabular}

$\begin{array}{ll}\text { SCANS } & 32 \\ \text { ACQTM } & 3.033 \mathrm{sec}\end{array}$

$\begin{array}{lr}\text { PD } & 3.967 \mathrm{sec} \\ \text { PW1 } & 5.0 \mathrm{us}\end{array}$

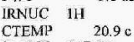

SLVNT CSDSN
EXREF 7.19 ppI

$\begin{array}{ll}\text { BF } & 1.20 \\ \text { RGAIN } & 16\end{array}$

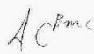

0310220

HMR spectrum of $\mathbf{1 b}$

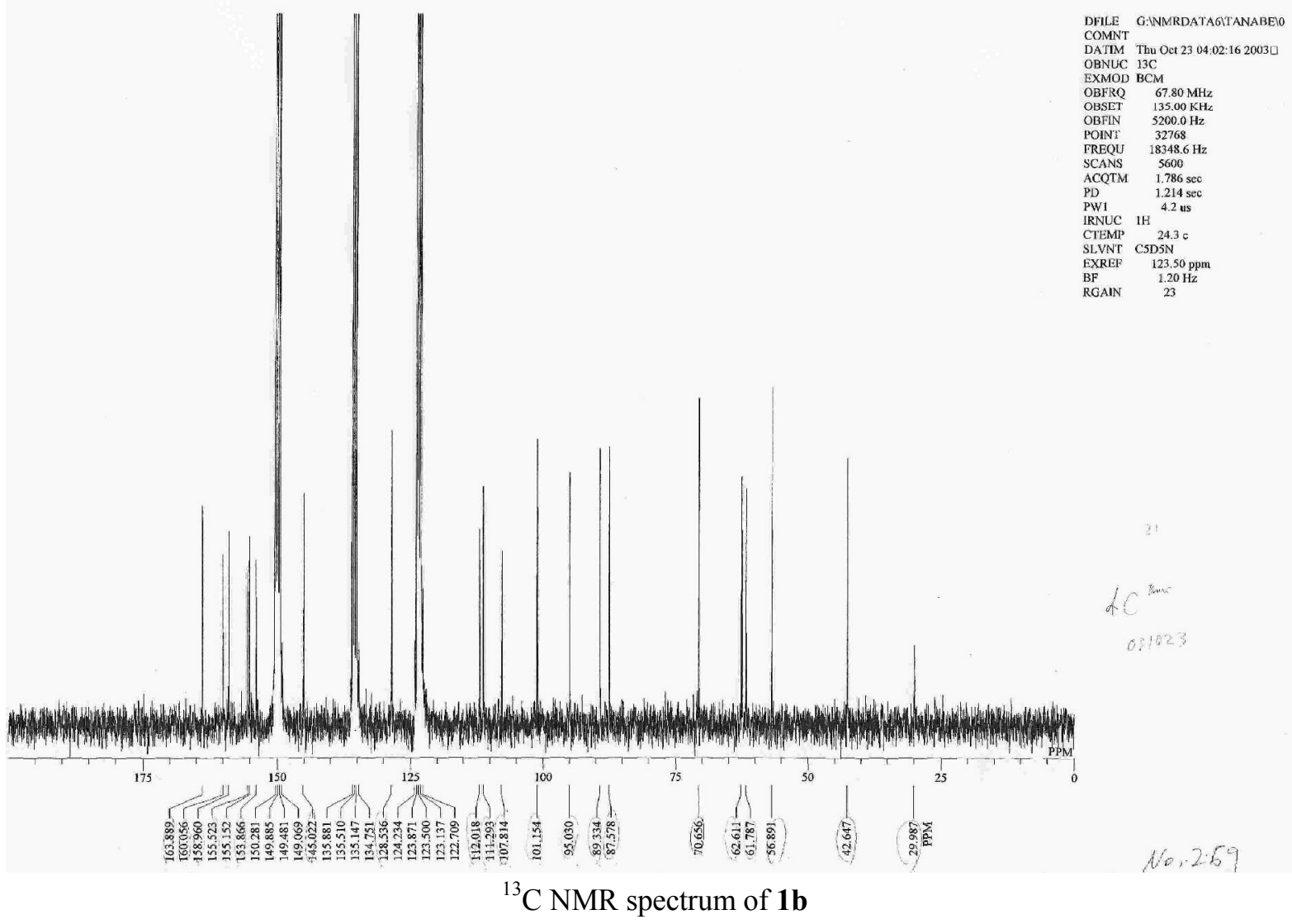




\section{4- $N$-(6-Nitrovertryloxycarbonyl) -2'-deoxycytidine (NVOC-dC, 1c)}

Compound 1c was prepared as reported by Alvarez et $\mathrm{al}^{1}$. Starting from $299.8 \mathrm{mg}(1.13 \mathrm{mmol})$ of 2'-deoxycytidine hydrochloride and $377 \mathrm{mg}$ (1.37 mmol) of NVOC-Cl, $205.6 \mathrm{mg}$ (0.44 mmol, 39\% yield) of $1 \mathbf{c}$ was obtained.

${ }^{1} \mathrm{H}$ NMR (pyridine-d $\left.\mathrm{d}_{5}\right) \delta 8.85(1 \mathrm{H}, \mathrm{d}, \mathrm{J}=7.5 \mathrm{~Hz}), 7.77(1 \mathrm{H}, \mathrm{s}), 7.44(1 \mathrm{H}, \mathrm{d}, \mathrm{J}=7.5 \mathrm{~Hz}), 7.21(1 \mathrm{H}, \mathrm{s}), 6.91$ $(1 \mathrm{H}, \mathrm{dd}, \mathrm{J}=6$ and $6 \mathrm{~Hz}), 5.85(2 \mathrm{H}, \mathrm{s}), 5.03(3 \mathrm{H}, \mathrm{brs}), 5.00(1 \mathrm{H}, \mathrm{m}), 4.51(1 \mathrm{H}, \mathrm{m}), 4.22(1 \mathrm{H}, \mathrm{dd}, \mathrm{J}=12$ and 3 $\mathrm{Hz}), 4.15(1 \mathrm{H}, \mathrm{dd}, \mathrm{J}=12$ and $3 \mathrm{~Hz}), 3.77(3 \mathrm{H}, \mathrm{s}), 3.62(3 \mathrm{H} . \mathrm{s}), 2.95(1 \mathrm{H}, \mathrm{ddd}, \mathrm{J}=13,5.5$ and $5.5 \mathrm{~Hz}), 2.62$ $(1 \mathrm{H}, \mathrm{ddd}, \mathrm{J}=13,6$ and $6 \mathrm{~Hz})$

${ }^{13} \mathrm{C}$ NMR (pyridine-d $\left.\mathrm{d}_{5}\right) \delta 164.0$ (s), 155.6 (s), 154.3 (s), 154.1 (s), 148.9 (s), 144.9 (d), 140.3 (s), 127.4 (s), 111.1 (d), 108.8 (d), 95.0 (d), 89.3 (d), 87.6 (d), 70.7 (d), $64.4(\mathrm{t}), 61.8(\mathrm{t}), 56.1(\mathrm{q}), 56.1(\mathrm{q}), 42.6(\mathrm{t})$.

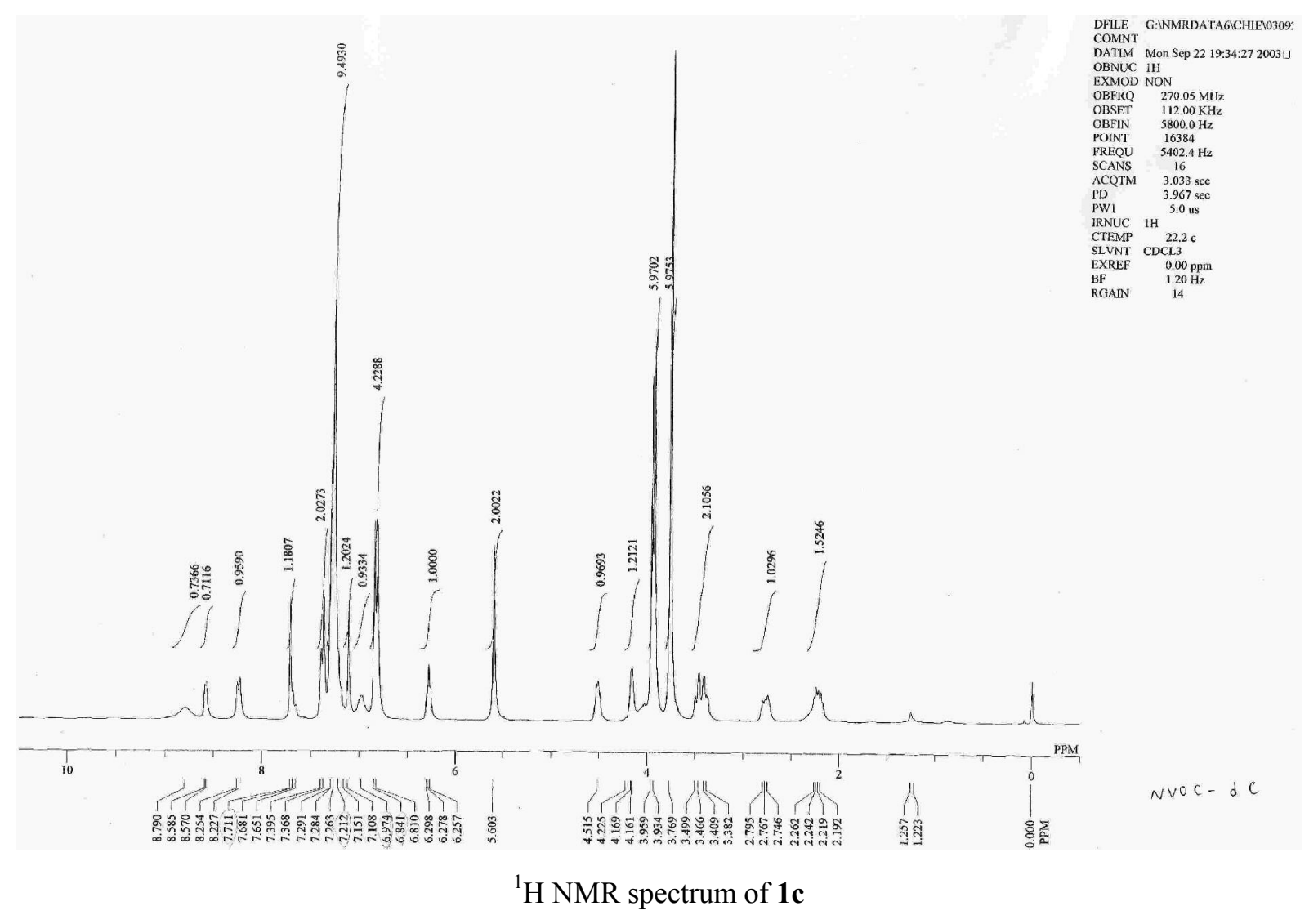




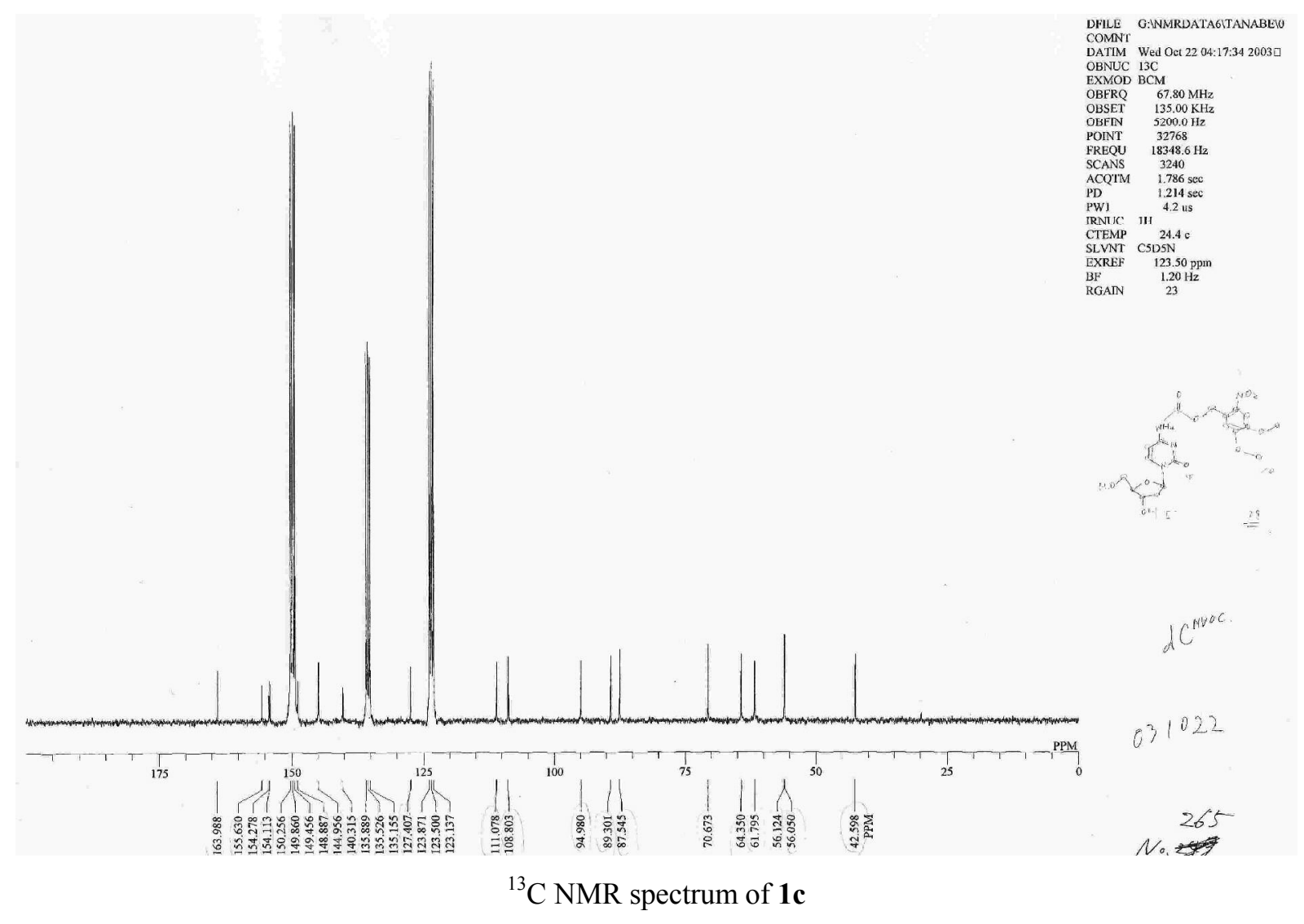

\section{4- $N$-[2-(2-nitrophenyl)propyloxycarbonyl]-2'-deoxycytidine (NPPOC-dC, 1d)}

Compound 1d was prepared following the procedure described for $\mathbf{1 b}$, starting from $264.1 \mathrm{mg}(1.00 \mathrm{mmol})$ of 2'-deoxycytidine hydrochloride and $311.2 \mathrm{mg}$ (1.28 mmol) of 2-(2-nitrophenyl)propyl chloroformate. ${ }^{2}$ Chromatographic purification ( $40 \mathrm{~g}$ of Silica gel $60, \mathrm{CHCl}_{3} / \mathrm{CH}_{3} \mathrm{OH}=15 / 1$ as an eluent) gave $429.9 \mathrm{mg}$ ( $0.990 \mathrm{mmol}, 99 \%$ yield) of $\mathbf{1 d}$.

${ }^{1} \mathrm{H}$ NMR $\left(\mathrm{CD}_{3} \mathrm{OD}\right) \delta 8.42(1 \mathrm{H}, \mathrm{d}, \mathrm{J}=7.5 \mathrm{~Hz}), 7.78(1 \mathrm{H}, \mathrm{d}, \mathrm{J}=7.5 \mathrm{~Hz}), 7.64(1 \mathrm{H}, \mathrm{m}), 7.63(1 \mathrm{H}, \mathrm{s}), 7.43(1 \mathrm{H}$, m), 7.24-7.13 (2H, m), $6.21(1 \mathrm{H}, \mathrm{dd}, \mathrm{J}=6$ and $6 \mathrm{~Hz}), 4.45-4.35(3 \mathrm{H}, \mathrm{m}), 4.01(1 \mathrm{H}, \mathrm{dd}, \mathrm{J}=3$ and $3 \mathrm{~Hz}), 3.85$ $(1 \mathrm{H}, \mathrm{dd}, \mathrm{J}=12$ and $3 \mathrm{~Hz}), 3.76(1 \mathrm{H}, \mathrm{dd}, \mathrm{J}=12$ and $3.5 \mathrm{~Hz}), 3.69(1 \mathrm{H}, \mathrm{q}, \mathrm{J}=7 \mathrm{~Hz}), 2.50(1 \mathrm{H}, \mathrm{ddd}, \mathrm{J}=13,6$ and $4.5 \mathrm{~Hz}), 2.17(1 \mathrm{H}, \mathrm{ddd}, \mathrm{J}=13,6.5$ and $6.5 \mathrm{~Hz}), 1.39(3 \mathrm{H}, \mathrm{d}, \mathrm{J}=7 \mathrm{~Hz})$

${ }^{13} \mathrm{C} \mathrm{NMR}\left(\mathrm{CDCl}_{3}\right) \delta 162.7$ (s), 155.8 (s), 152.6 (s), 150.2 (s), 144.8 (d), 136.6 (s), 132.7 (d), 128.1 (d), $127.6(\mathrm{~d}), 124.1$ (d), 95.5 (d), 87.5 (d), 87.5 (d), 69.7 (d), 69.5 (t), $61.2(\mathrm{t}), 41.1(\mathrm{t}), 33.1(\mathrm{~d}), 17.6(\mathrm{q})$ FT-IR (neat) 3100-3500 (brd), 2918, 1752, 1717, 1700, 1683, 1658, 1649, 1642, 1631, 1564, 1524, 1501, 1202, 1092, 1062, $998 \mathrm{~cm}^{-1}$.

ESI-MS (positive) $\mathrm{m} / \mathrm{z} 435\left(\mathrm{C}_{19} \mathrm{H}_{22} \mathrm{~N}_{4} \mathrm{O}_{8}+\mathrm{H}^{+}\right)$ 


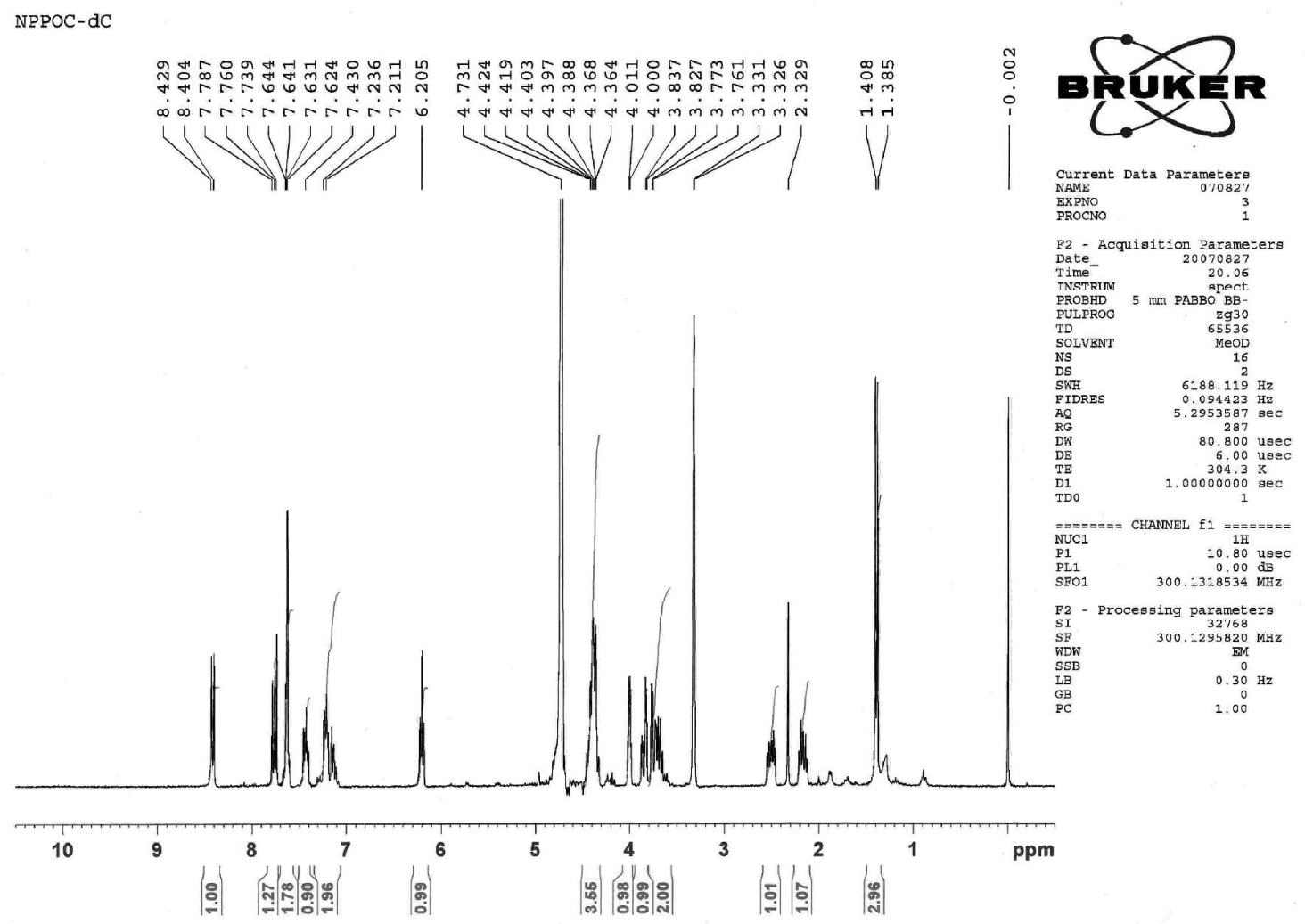

${ }^{1} \mathrm{H}$ NMR spectrum of $\mathbf{1 d}$

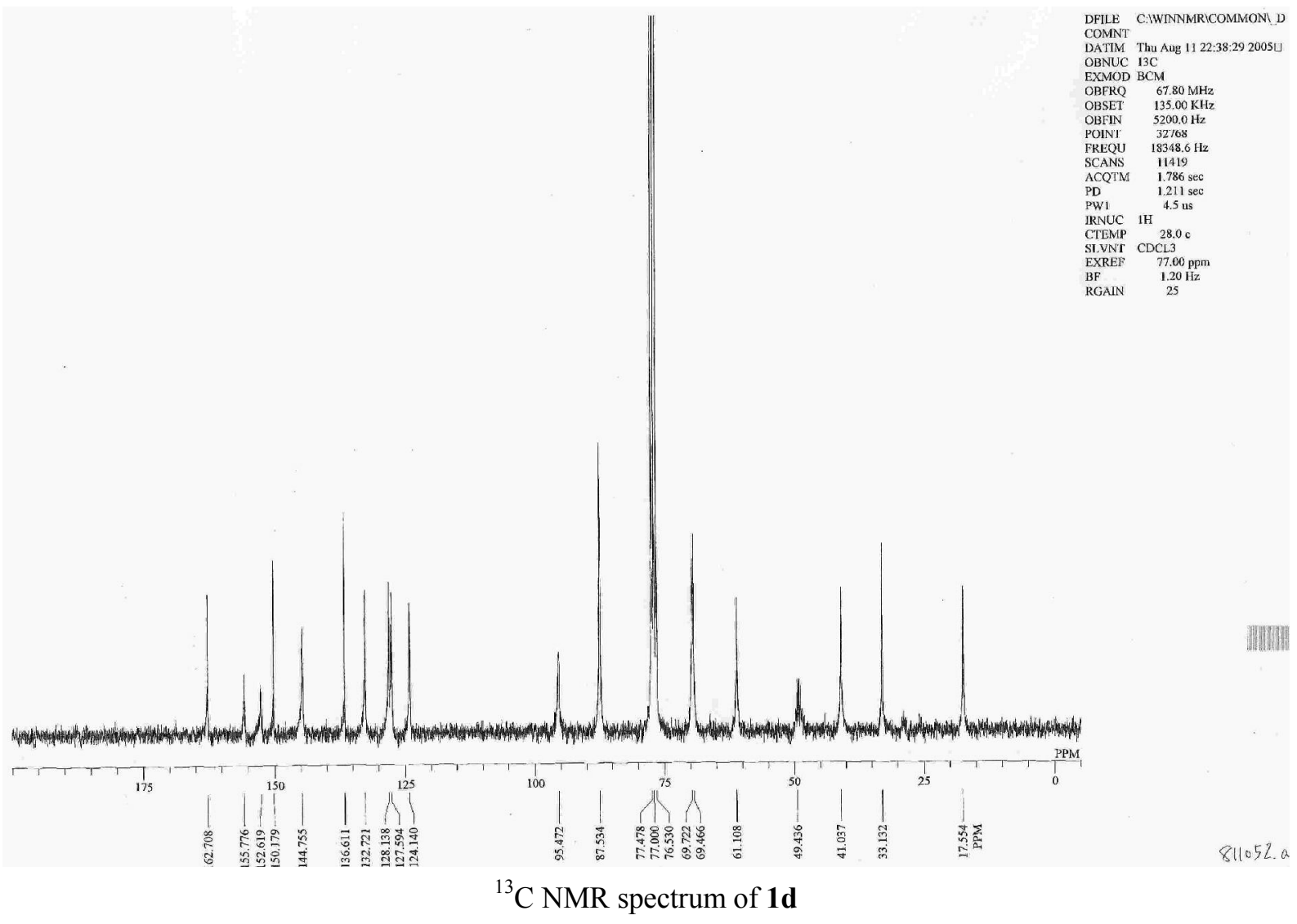




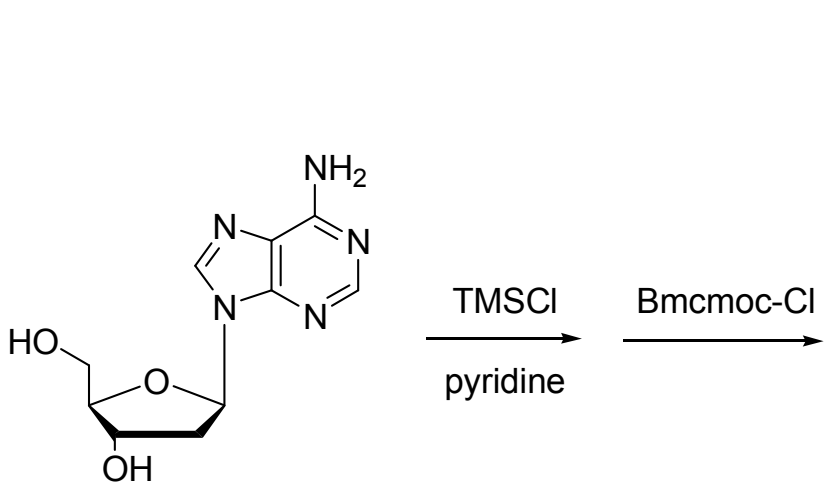

2'-deoxyadenosine<smiles>COc1cc2oc(=O)cc(COC(=O)Nc3ncnc4c3ncn4C3CC(O)C(CO)O3)c2cc1Br</smiles>

2b: Bmcmoc-dA

Compound $\mathbf{2 b}$ was prepared following the procedure described for $\mathbf{1 b}$. Briefly, $302.9 \mathrm{mg}$ (1.21 mmol) of 2'-deoxyadenosine in dry pyridine $(10 \mathrm{~mL})$ was reacted with $1 \mathrm{~mL}(7.82 \mathrm{mmol})$ of $\mathrm{TMSCl}$ and then $482 \mathrm{mg}$ (1.39 mmol) of Bmcmoc-Cl to yield $21.0 \mathrm{mg}$ ( $35.6 \mu \mathrm{mol}, 2.9 \%$ yield) of $\mathbf{2 b}$.

${ }^{1} \mathrm{H}$ NMR (pyridine-d $\left.\mathrm{d}_{5}\right) \delta 9.03(1 \mathrm{H}, \mathrm{s}), 9.01(1 \mathrm{H}, \mathrm{s}), 8.08(1 \mathrm{H}, \mathrm{s}), 6.99(1 \mathrm{H}, \mathrm{dd}, \mathrm{J}=6.5$ and $6.5 \mathrm{~Hz}), 6.96(1 \mathrm{H}$, s), , $6.83(1 \mathrm{H}, \mathrm{s}), 5.75(2 \mathrm{H}, \mathrm{s}), 5.21(1 \mathrm{H}, \mathrm{m}), 5.03(2 \mathrm{H}, \mathrm{brs}), 4.60(1 \mathrm{H}, \mathrm{m}), 4.23(1 \mathrm{H}, \mathrm{dd}, \mathrm{J}=11$ and $4 \mathrm{~Hz})$, $4.15(1 \mathrm{H}, \mathrm{dd}, \mathrm{J}=11$ and $3 \mathrm{~Hz}), 3.82(1 \mathrm{H}, \mathrm{m}), 3.79(3 \mathrm{H}, \mathrm{s}), 3.16(1 \mathrm{H}, \mathrm{ddd}, 13,6.5$ and $6.5 \mathrm{~Hz}), 2.82(1 \mathrm{H}$, ddd, $\mathrm{J}=13,6.5$ and $4 \mathrm{~Hz}$ ).

${ }^{13} \mathrm{C}$ NMR (pyridine-d $\mathrm{d}_{5}$ ) $\delta 160.2(\mathrm{~s}), 158.8$ (s), 155.1 (s), 153.3 (s), 152.7 (s), 152.4 (d), 152.2 (s), 150.7 (s), 142.9 (d), 128.6 (d), 124.2 (s), 112.1 (s), 111.4 (d), 107.7 (s), 101.1 (d), 89.7 (d), 85.4 (d), 71.7 (d), 62.8 (t), $62.7(\mathrm{t}), 56.8(\mathrm{q}), 41.4(\mathrm{t})$

FT-IR (neat) 3200-3500 (brd), 2962, 1737, 1726, 1711, 1605, 1259, 1208, 1156, 1092, 1027, $798 \mathrm{~cm}^{-1}$. 


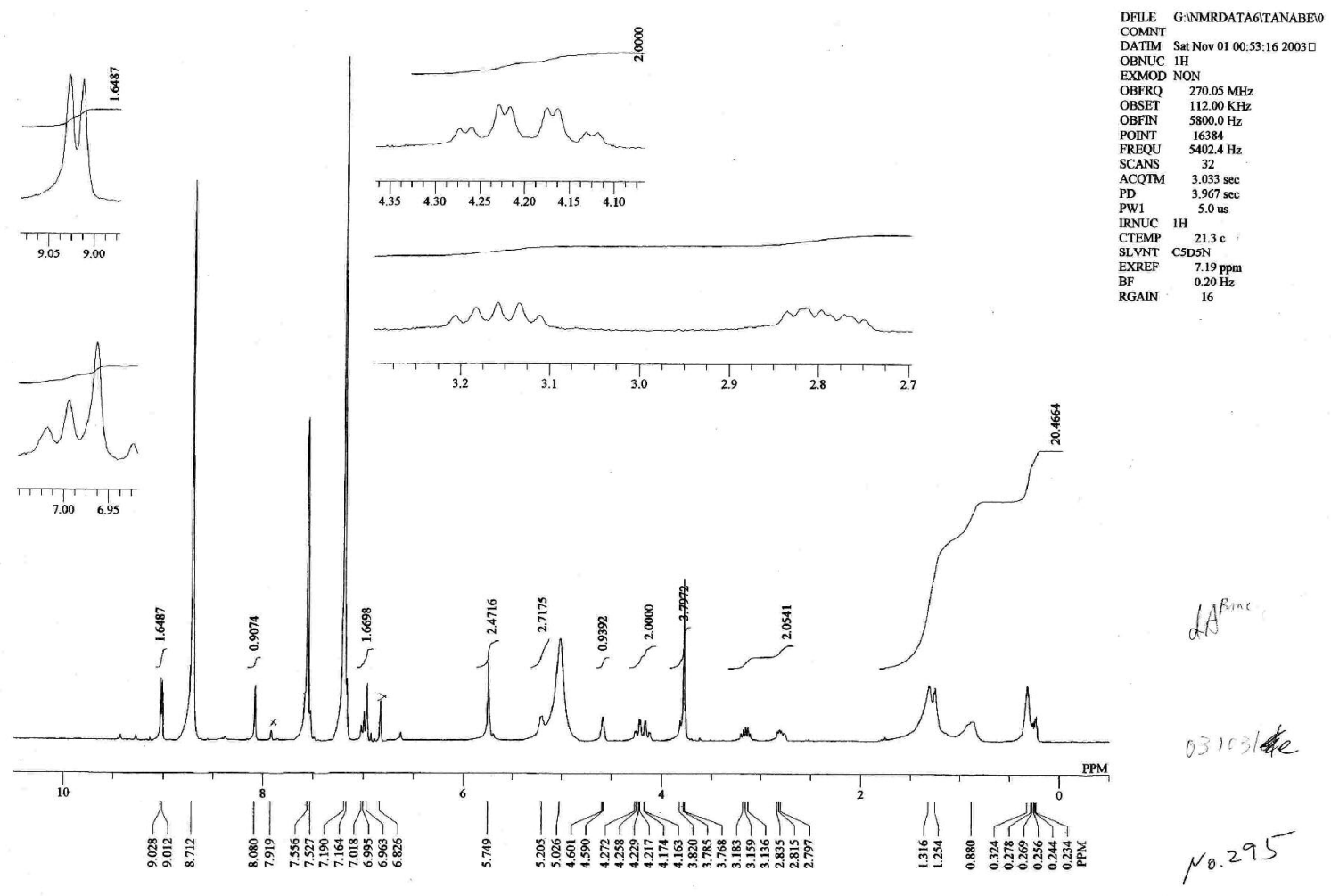

${ }^{1} \mathrm{H}$ NMR spectrum of $\mathbf{2 b}$

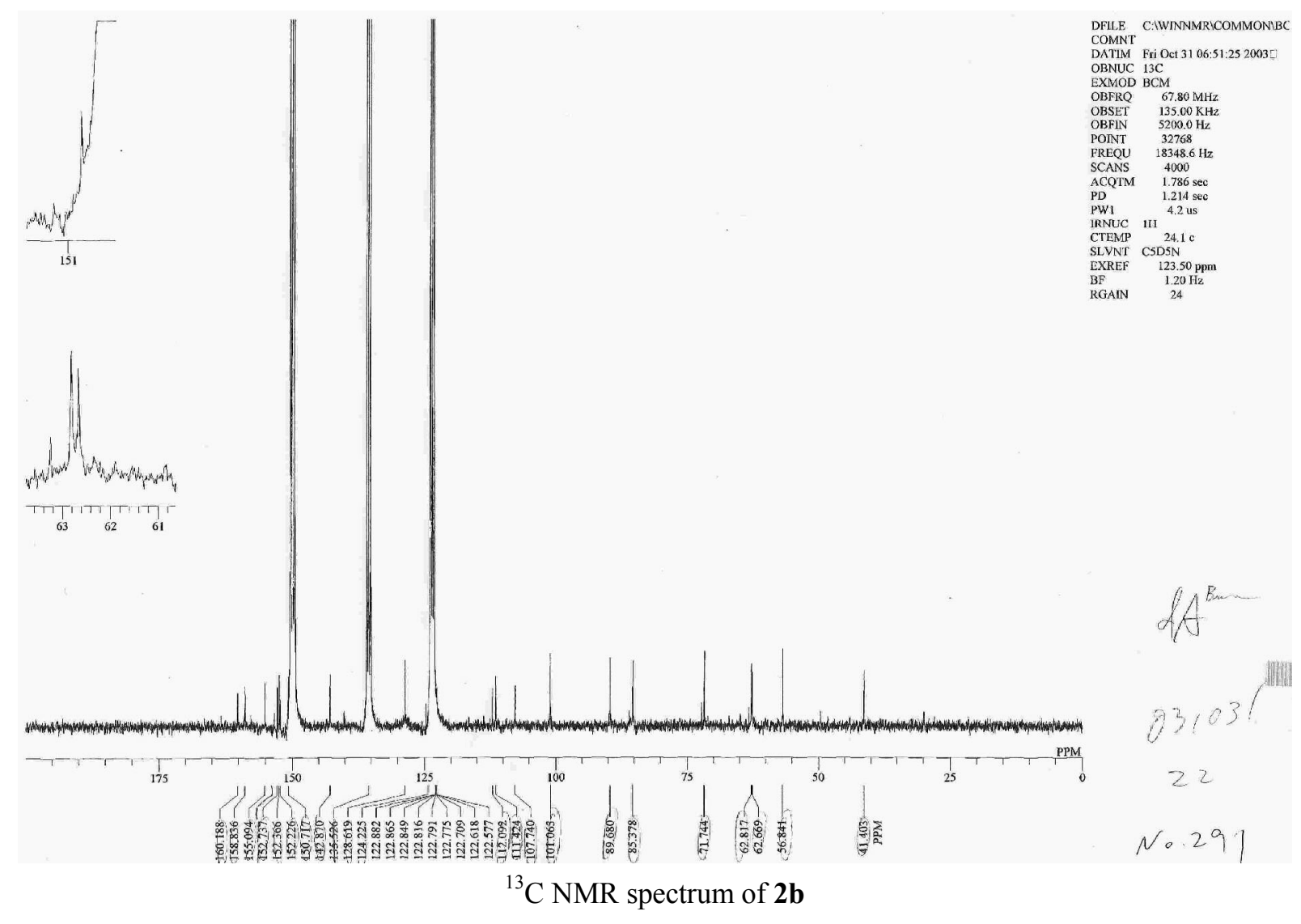


<smiles>[R6]C[C@]1(O)O[C@@H]2C[C@@H]1O[C@H]2n1cnc2c(N)ncnc21</smiles>
$\mathrm{dA} / \mathrm{TBS}(\mathrm{R}=\mathrm{TBDMS})$ 2'-deoxyadenosine $(\mathrm{R}=\mathrm{H})$

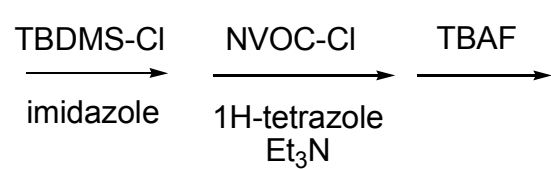

Compound 2c was prepared following the reported procedure by Alvarez et al ${ }^{1}$.

${ }^{1} \mathrm{H}$ NMR (pyridine- $\left.\mathrm{d}_{5}\right) \delta 9.03(1 \mathrm{H}, \mathrm{s}), 8.97(1 \mathrm{H}, \mathrm{s}), 7.77(1 \mathrm{H}, \mathrm{s}), 7.59(1 \mathrm{H}, \mathrm{s}), 6.99(1 \mathrm{H}, \mathrm{dd}, \mathrm{J}=6.5$ and 6.5 $\mathrm{Hz}), 6.01(2 \mathrm{H}, \mathrm{s}), 5.62(2 \mathrm{H}, \mathrm{brs}), 5.21(1 \mathrm{H}, \mathrm{brs}), 4.59(1 \mathrm{H}, \mathrm{m}), 4.23(1 \mathrm{H}, \mathrm{dd}, \mathrm{J}=12$ and $3 \mathrm{~Hz}), 4.15(1 \mathrm{H}, \mathrm{dd}$, $\mathrm{J}=12$ and $3 \mathrm{~Hz}), 3.82(1 \mathrm{H}, \mathrm{m}), 3.76(3 \mathrm{H}, \mathrm{s}), 3.75(3 \mathrm{H}, \mathrm{s}), 3.15(1 \mathrm{H}, \mathrm{ddd}, \mathrm{J}=13,6.5$ and $6.5 \mathrm{~Hz}), 2.80(1 \mathrm{H}$. $\mathrm{m})$.

${ }^{13} \mathrm{C}$ NMR (pyridine- $\mathrm{d}_{5}$ ) $\delta 154.5$ (s), 153.2 (d), 152.4 (s), 152.1 (s), 150.9 (s), 148.7 (s), 142.8 (d), 128.3 (s), $124.6(\mathrm{~s}), 111.0(\mathrm{~d}), 108.7$ (d), 89.7 (d), $85.4(\mathrm{~d}), 71.8(\mathrm{~d}), 64.7(\mathrm{t}), 62.7(\mathrm{t}), 56.2(\mathrm{q}), 56.1(\mathrm{q}), 41.4(\mathrm{t})$

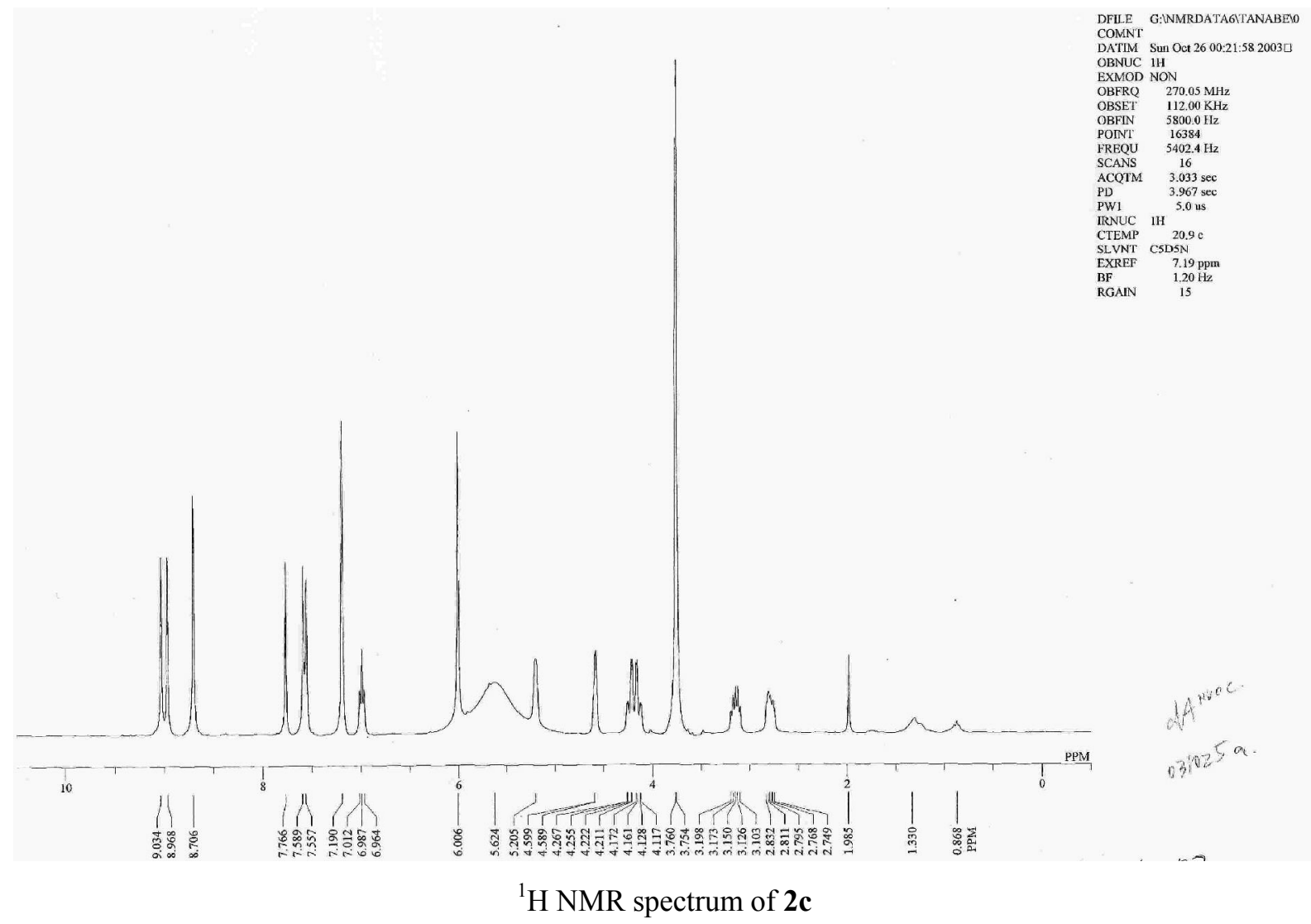




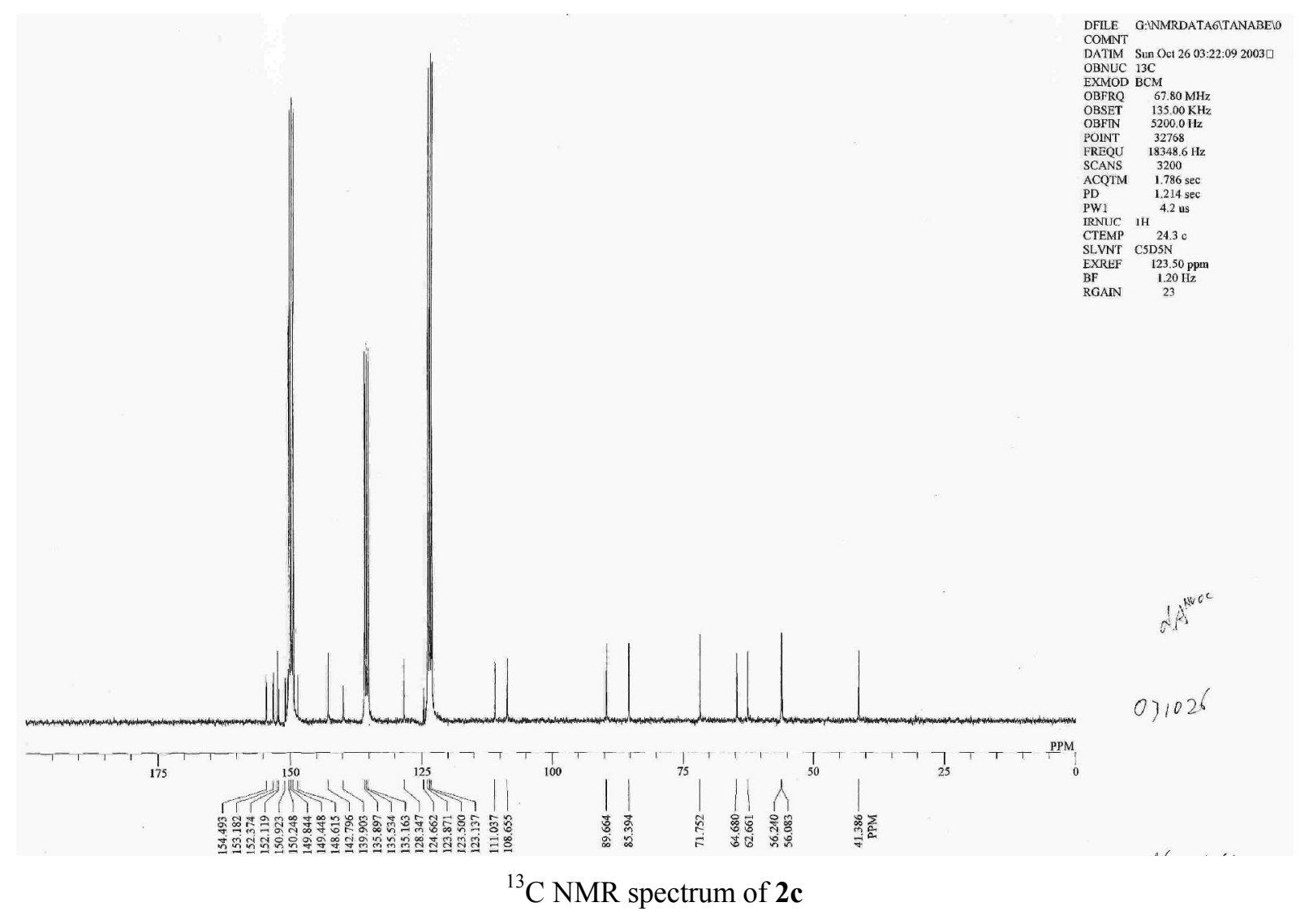




\section{Photolysis and quantum efficiency measurement-}

Into a pyrex test tube of $12 \mathrm{~mm}$ diameter was placed $2 \mathrm{~mL}$ of $10 \mu \mathrm{M}$ substrate solution in K-MOPS solution ( $\mathrm{pH}$ 7.2) containing 0.1\% DMSO. The solution was irradiated at $350 \mathrm{~nm}$ using two RPR $350 \mathrm{~nm}$ lamps $\left(10 \mathrm{~mJ} \mathrm{~s}^{-1}\right)$. Aliquots of $10 \mu \mathrm{L}$ were removed periodically and analyzed by HPLC. The light output for the quantum efficiencies measurement was performed using ferrioxalate actinometry. ${ }^{3}$

HPLC traces for the photolysis of $\mathbf{1 b}$ is shown in Fig. S1: Cosmosil 5C18 AR II (250 X 4 mm, Nacalai), $1.0 \mathrm{~mL} / \mathrm{min}, 5-45 \% \mathrm{CH}_{3} \mathrm{CN}$ in $\mathrm{H}_{2} \mathrm{O}(0-10 \mathrm{~min})$, detection at $260 \mathrm{~nm}$. From the HPLC traces (Fig. 3 in main text and Fig S1), the released dC from 1a and $\mathbf{1 b}$ was quantified and plotted against the irradiation time (Fig. S2).

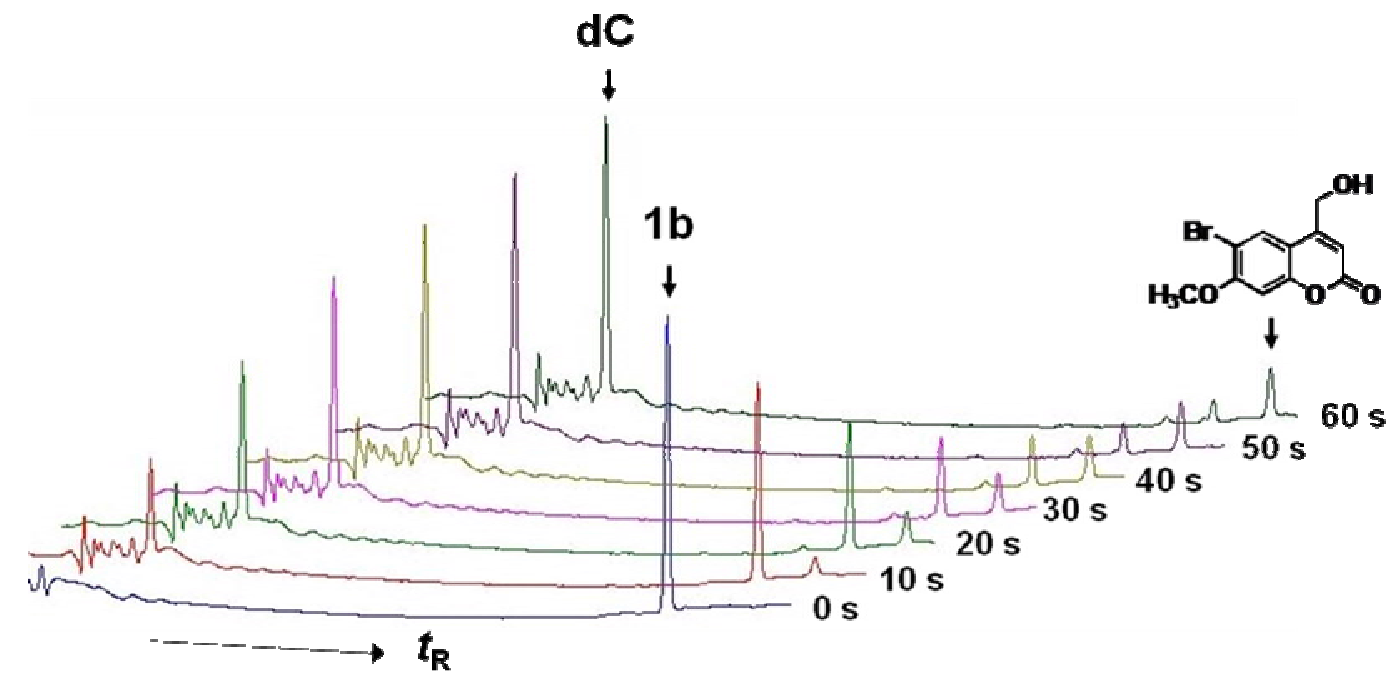

Figure S1. HPLC traces for the photolysis of $\mathbf{1 b}$.

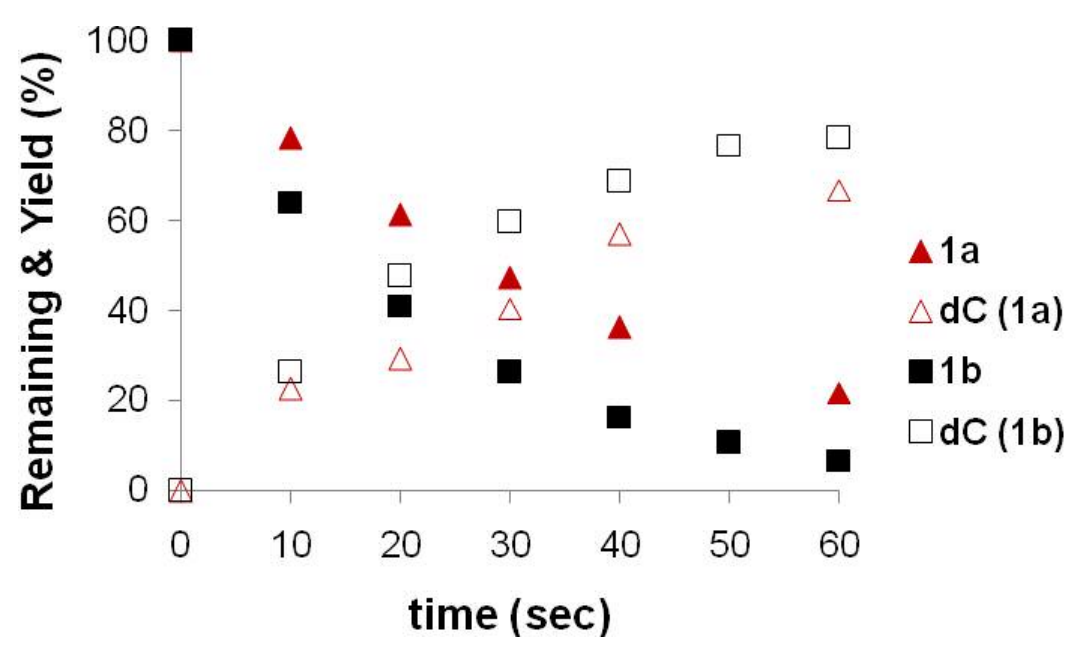

Figure S2. Time course for the photolysis of $\mathbf{1 a}$ and $\mathbf{1 b}$. Samples $\left(10^{-5} \mathrm{M}\right)$ were irradiated at $350 \mathrm{~nm}(10$ $\mathrm{mJ} / \mathrm{s}$ ) under simulated physiological conditions. 1a (red, triangle), 1b (black, square), dC from 1a (red, open triangle), $\mathrm{dC}$ from $\mathbf{1 b}$ (black, open square). 


\section{Measurements of two-photon uncaging action cross sections-}

Measurements were carried out in microcuvetts with $10 \times 1 \times 1 \mathrm{~mm}$ illuminated dimensions and $15 \mu \mathrm{L}$ filling volume (16.10F-Q-10; Starna, Atascadero, CA). Femtosecond near IR pulses from a mode-locked, Ti-sapphire laser (Tsunami pumped by Millenium V; Spectra-Physics) were focused on the center of the sample chamber (10 mm path length) with a $25 \mathrm{~mm}$ focal length lens (06LXP003/076; Melles Griot, Irvine, CA) optimized for IR lasers. The two-photon uncaging action cross-section $\left(\delta_{u}\right)$ was estimated by referencing to fluorescein, whose fluorescence quantum yield $\Phi_{\mathrm{F}}(0.9)$ and two-photon absorption cross-section $\delta_{\mathrm{aF}}$ (22 and $30 \mathrm{GM}$ at 720 and $740 \mathrm{~nm}$, respectively) were characterized in the literature. ${ }^{4}$

The two-photon uncaging action cross-section is given by the equation

$$
\delta_{\mathrm{u}}=\left(\mathrm{N}_{\mathrm{p}} \mathrm{K} \Phi_{\mathrm{F}} \delta_{\mathrm{aF}} \mathrm{C}_{\mathrm{F}}\right) /\left(<\mathrm{F}(\mathrm{t})>\mathrm{C}_{\mathrm{S}}\right)
$$

where $N p$ is the number of product molecules formed per unit time (molecules/s, determined by HPLC analysis); $\mathrm{K}$ is the collection efficiency of our experimental setup used to measure the fluorescence of fluorescein emitted at a right angle to the beam and passed through a $520 \pm 10 \mathrm{~nm}$ bandpass filter (03FIV109, Melles Griot, Irvine, CA); $C_{\mathrm{F}}$ is the concentration of fluorescein $(\mathrm{mol} / \mathrm{L}) ;<F(t)>$ is the time averaged fluorescence (photons/s) collected by the detector (silicon photodiode radiometer, SED033 on an IL-1700, International Light, Newburyport, MA); and $C_{\mathrm{S}}$ is the initial concentration of the caged substrate $(\mathrm{mol} / \mathrm{L})$.

\section{Reference}

(1) Alvarez, K.; Vasseur, J. J.; Beltran, T.; Imbach, J. L. J. Org. Chem. 1999, 64, 6319.

(2) Hasan, A.; Stengele, K-P.; Giegrich, H.; Cornwell, P.; Isham, K. R.; Sachleben, R. A.; Pfleiderer, W.; Foote, R. S. Tetrahedron, 1997, 53, 4247.

(3) Hatchard, C. G.; Parker, C. A. Proc. R. Soc. London Ser. A 1956, 235, 518.

(4) Xu,C.; Web, W. W. J. Opt. Soc. Am. B, 1996, 13, 481. 